\title{
The Role of Hip Arthroscopy in the Diagnosis and Management of Early Avascular Necrosis: A Case Report

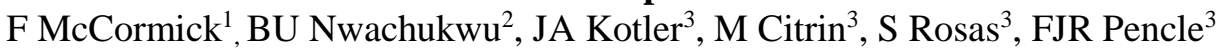

\begin{abstract}
Objective: The management of pre-collapse osteonecrosis of the femoral head is controversial. Core decompression is a technique that theoretically decreases the intraosseos pressure of the femoral head, and is appropriate for Ficat Stage II AVN, and in a small proportion of patients at the transition from stage II to III. Arthroscopic evaluation can aid in staging femoral head AVN in cases in which X-ray and MRI findings are equivocal. We describe the technique of arthroscopic evaluation to aid in staging of femoral head osteonecrosis, and core-decompression of the femoral head.
\end{abstract}

Case Report: We present a case of AVN in a young SLE patient on chronic high-dose corticosteroid therapy, who we found appropriate for arthroscopic evaluation and treatment with core decompression due to inconclusive X-ray and MRI findings.

Conclusion: The advantages of arthroscopic evaluation and treatment include the ability to evaluate by direct visualization the articular surface for the presence or absence of articular cartilage injury, subchondral collapse, and guidance during reaming and curettage. It also allows the ability to address and concomitant soft-tissue or bony pathology associated with or in addition to the osteonecrotic lesion.

Keywords: Arthroscopy, avascular necrosis, equivocal test, MRI, staging, X-Ray

From: ${ }^{1}$ The Spine and Orthopedic Academic Research Institute (SOAR), 5511 North Dixie Highway 7th Street, Fort Lauderdale, FL, 33062, ${ }^{2}$ Department of Orthopedic Surgery, Hospital for Special Surgery, 535 East 70th Street, New York NY, 10021, ${ }^{3}$ Holy Cross Orthopedic Research Institute, 4595 North Dixie Highway, Fort Lauderdale, FL, 33308.

Correspondence: Dr F PencleHoly Cross Orthopedic Research Institute 4595 North Dixie Highway, Fort Lauderdale, FL, 33308, USA.Email: fabio.pencle@gmail.com 


\section{INTRODUCTION}

Avascular necrosis (AVN) of the femoral head is a musculoskeletal pathology resulting from a reduction of blood supply to the femoral head.(1-4) AVN is characterized by areas of dead trabecular bone and marrow extending to include the subchondral plate. The most common causes of AVN are trauma and chronic use of exogenous steroids $(3,5,6)$. In these scenarios, revascularization is limited and the femoral head progresses to eventual subchondral collapse if not treated with core decompression $(7,8)$.

The impact of AVN is being increasingly reported and this condition has become an increasingly common cause of musculoskeletal disability, accounting for 10,000-20,000 total hip replacements in young patients per year in the US $(8,9)$ Corticosteroid-induced vascular change in bone is a significant cause of AVN. High dose corticosteroid regimens are a mainstay of treatment in patients with a history of Systemic Lupus Erythematosus (SLE) and this patient population is at increased risk of $\mathrm{AVN}$, and fast progression of disease. (9)

Treatment of early AVN focuses on preventing progression of the disease and eventual subchondral collapse. Because positive outcomes are observed in patients that receive core decompression at the transition from mild to moderate disease the efficacy of the procedure is limited by the physician's ability to accurately identify the stage of disease. The Ficat classification system for staging AVN of the hip relies upon plain film, MRI, and clinical features to assign disease stages ranging from 0-4 (See Table I and Table II). Stage IV is marked by complete collapse of the femoral head and indicates a total or hemi hip arthroplasty; Stage III AVN is treated similarly with the option of osteotomy in lieu of joint arthroplasty depending on the extent of involvement(10). Surgical decompression is often successful if performed at Stage I or II of disease (10). As Stage II begins to 
transition to Stage III the femoral head demonstrates a "crescent sign" on plain film and MRI with marked pain and stiffness radiating to the knee. Core decompression performed at or before this time can prevent complete subchondral collapse. (11) Even with the use of radiographic imaging, difficulty identifying the stage of the disease prevents appropriate treatment with core decompression at the optimal point in disease progression. Magnetic resonance imaging is considered the most sensitive non-invasive approach for differentiating stages of early AVN. Hip arthroscopy is associated with minimal morbidity and could be potentially used to more accurately evaluate AVN for core decompression. Arthroscopic diagnosis may be particularly useful for patients with risk factors for fast progression, or who have mechanical symptoms that can be concomitantly addressed.

\section{CASE REPORT}

A 38-year-old African-American female with history of SLE presented with intractable right hip pain during weight bearing that had persisted for approximately 6 months. Her pain was predominantly in her groin and was non-radiating in nature. She required crutches to ambulate and had failed conservative treatments prescribed by her primary physician. Conservative measures had included ice, NSAIDs, physical therapy and pain relievers. A review of systems was only remarkable for musculoskeletal related hip pathology. Due to medical history of SLE first diagnosed at age 22 , she had been on long-term chronic oral corticosteroid therapy for 16 years. Recent addition of Plaquenil (Hydroxychloroquine sulfate, Covis, NC, USA) has enabled her to reduce her daily corticosteroid dosage of Prednisone from $20 \mathrm{mg}$ to $5 \mathrm{mg}$. She had no other medical problems or prior surgeries. She was a non-smoker, did not use illicit drugs but consumed alcohol 
in moderate amounts. Examination she had pain with hip flexion and internal rotation, pain with weight bearing, full passive range of motion, and normal strength assessment. On strength testing, there was equal strength bilaterally and she was neurovascularly intact in her distal extremities.

For imaging, four plain radiographic views of the hip/pelvis were obtained which included AP Pelvis, Oblique, true lateral, and frog-leg lateral (Figures 1, 2). Plain radiographs demonstrated a concentric joint with maintenance of her joint spaces, mild subchondral sclerotic changes without collapse or crescent sign (Figure 1,2). There was no dysplasia or significant cam or pincer impingement lesions. Standard T1, T2, and short T1 inversion Recovery Magnetic Resonance Imaging(MRI) contrast demonstrated osteonecrosis in the anterosuperior surface of the femoral head, with evidence of subcortical edema, and without evidence of subchondral collapse or crescent sign (Figure 3,4 and 5). No other findings where present in the pre-operative MRI such as labral tears or loose bodies (Figure 4, 5).

Patient was interested in undergoing a right hip arthroscopy to ensure there was no subchondral collapse. If that were the case we would perform a planned core decompression of the affected area, using arthroscopic and fluoroscopic guidance. We also anticipated aspiration of bone marrow to pack the hip and also planned active healing to bone. Of note, if she was found to not be a candidate for core decompression at the time of surgery, we will stage a subchondral collapse in anticipation for osteochondral allograft, given her young age. We will also consider arthroscopic debridement. Because of persistent pain, and failed conservative management, the patient underwent hip arthroscopy and core decompression.

Hip arthroscopy was performed to evaluate the extent of osteonecrosis in the setting of inconclusive early stage MRI and X-ray findings. Of note there was no evidence of collapse; the articular surface was pristine upon arthroscopic visual inspection, with presence of significant 
synovitis in the anterior superior region, the synovium extending to the labrum. There was no articular wear to the acetabulum.

Then a reamer was used to perform the core decompression under fluoroscopic guidance as planned with the use of the arthroscope to confirm the placement of the reamer beneath the cartilage. The surgeon (F.M) noted during reaming the presence of highly sclerotic bone in the area of the subchondral plate, which might indicate the progression of the disease. The core decompression was finalized by utilizing packed bone chips and plasma concentrate to allow for proper hemostasis and bone structure (Figure 7).

\section{Procedure in detail}

The patient was positioned on the traction table with care taken to pad any bony prominences; Examination under anesthesia was performed without significant findings. Traction was induced under fluoroscopic guidance and confirmation of appropriate joint distraction. The hip was then prepped and draped in the usual fashion and a surgical time-out was performed. A right diagnostic hip arthroscopy was performed with verification of antibiotics as well as DVT prophylaxis on the non-operative extremity. This was begun in the anterolateral approach under fluoroscopic guidance.

Next, the anterior approach was done under direct visualization. An inner portal capsulotomy was performed for instrumentation. There was found to be significant synovitis and irritation in the anterior and superior aspect of the synovium as well as the inferior aspect of the labrum. This was debrided using a rotary shaver and electrocautery device (29862). Next a separate surgical incision of bone graft was taken for harvesting into the hip at a later date (20900). This was done using a sharp $11 \mathrm{~mm}$ probe. Approximately $50 \mathrm{~mL}$ of fluid was taken out as well as bone graft. Next (20900.59) a core decompression was done under direct visualization. This was 
done initially with a Steinman pin doing a center-center approach to the anterior superior aspect of the bone. Using fluoroscopic guidance, we took this down to the area of avascular necrosis to allow for decompression at the same time, ensuring no penetration into the joint using arthroscopic visualization. We then took a $6 \mathrm{~mm}$ reamer into this area to decorticate the bone. The bone was found to be hard and sclerotic. We took this down to as close to the cortices as felt safe. This was approximately $7 \mathrm{~mm}$ by estimate away from the subchondral region at its apex.

We confirmed that it did not breech the cortices arthroscopically as we had reamed. Next, after completing the reaming, the reamer was removed and bone graft and plasma concentrate was then injected into the defect at the area of the avascular necrosis and subchondral collapse (I think we should take out subchondral collapse here even though it was in the operative report). This was then packed with bone chips. Next, a bone graft was placed in the cortical defects to allow for minimized bleeding and compression. This was packed tightly. After completion of fluoroscopy, there was equivalent fluorescence of the bone indicating a tight pack of demineralized bone graft. The instruments were then removed from the patient.

The wounds were closed copiously and closed with suture layers. Sterile dressings were applied as well as compressive dressing and cryotherapy. The patient was extubated and taken to the PACU in stable condition. Post-operatively, the patient was allowed touch-down weight bearing with crutches for 6 weeks. Immediate passive range of motion began the evening of surgery. At 6 weeks the patient progressed to weight-bearing as tolerated. Return to athletic activity was delayed until three months. 3 months following surgery she presented for painful hip on the contralateral side, which we also evaluated and which demonstrated AVN which appeared more severe than the right hip she had originally presented for. 


\section{DISCUSSION}

The pathogenesis of $\mathrm{ON}$ of the femoral head is not fully understood, but is thought to be multifactorial, due to environmental and genetic factors. The most common risk factors for atraumatic AVN of the femoral head are high-dose corticosteroid usage and alcohol, and hypercoagulable states. Some chronic conditions are also commonly associated with an increased incidence of ON of the femoral head, including sickle-cell disease and SLE.

High-dose corticosteroid treatment is the most common risk factor for atraumatic Avascular Necrosis (AVN) of the femoral head $(1,2,12)$. Lupus is among the most commonly associated chronic disease, likely due in large part to necessitating treatment with chronic highdose corticosteroid regimens $(9,13-15)$, which increases risk for destructive vascular pathology(6, 9). The patient in this case study was treated with $20 \mathrm{mg}$ of Prednisone for almost 16 years due to SLE. She was decreased prior to surgery.

Additionally, this case revisits the question of using diagnostic hip arthroscopy in addition to traditional radiography to better discern pre-collapse. This is particularly appropriate in the setting of a chronic corticosteroid user in whom the disease process could progress with greater alacrity than radiographically detectable $(9,16)$, and also indicated as a means of evaluating and treating synovitis causing treatment recalcitrant hip pain.

Several treatment options exist for pre-collapse ON have been described, including core decompression, vascularized bone graft, cell-based treatment (stem cells, platelet rich plasma) (17, 18), and osteotomies $(16,19)$. The efficacy of such approaches has not been fully elucidated due to lack of prospective comparative data. In addition, vascularized bone graft requires a team of microsurgeons with significant experience(11). Rotational osteotomy also requires high-levels of 
experience and planning. Lack of experience with these techniques can lead to poor outcomes; therefore, core decompression remains an accepted treatment modality for patients with precollapse ON of the femoral head.

Over the past two decades, hip arthroscopy has evolved to where it is now the gold standard for the diagnosis of intra-articular joint pathology. Various advances in hip specific-radiography, MRI, and CT have assisted in the understanding of bony and soft-tissue pathology in patients with femoroacetabular impingement and hip dysplasia. The incidence and prevalence of soft-tissue and bony intra-and extra articular pathology is currently not known. In addition, the ability of radiography to predict collapse and/or associated hip pathology in addition to ON, at the time of surgery is also unknown. We therefore advocate that arthroscopy at the time of decompression provides the most accurate means to confirm the presence or absence of femoral head subchondral collapse, chondral delamination, and associated labral pathology. If present, these can be addressed at the time of decompression and obviate the need for a subsequent surgery. In addition, this will allow for verification and guidance during drilling and/or reaming preventing penetration of the articular surface.

Hip arthroscopy however, like all procedures has associated complications. A recent metaanalysis showed an overall $4 \%$ complication rate (20). Complications such as perineal skin breakdown, nerve palsy, infection, hematoma, and so on can all be minimized if the procedure is performed by an experienced surgeon(20-22). The risks and benefits of arthroscopy need to be weighed at the time of decompression. In the setting of a superimposed cam or pincer type femoroacetabular impingement, the benefit of combined arthroscopy and decompression may outweigh the risks. 
In summary, arthroscopically assisted core decompression of the femoral head for precollapse ON provides the added advantage of intra-articular visualization to confirm the diagnosis, allow for treatment of associated bony and soft-tissue pathology and avoid the risk of penetration in carefully selected patients.

The clinical utility of each Staging Systems lies in determining which hips are appropriate for intervention with Core decompression versus hip arthroplasty. Stage II disease responds favorably to core decompression with over $50 \%$ hip survivorship at five years in the largest metaanalysis (8). Stage III disease does not respond as well with hip survivorship of $37 \%$ of patients in the largest meta-analysis (5). There is also poor hip survivorship if cystic change is present as in late stage II disease(5). A shortcoming of simple radiographic staging is that disease can sometimes progress ahead of radiographic detection. MRI is perhaps the most sensitive radiographic method for detecting early disease (88-100\%), better than plain radiographs, CT, bone scanning $(23,24)$. However, in a study by Ruch et al. (2003) Arthroscopy of the hip revealed osteochondral degeneration that was not detected by plain radiographs or MRI in $36 \%$ of femoral heads.

Hip arthroscopy can enhance staging accuracy by allowing for mechanical testing of the joint surface and is minimally invasive. It was appropriate in our patient with stage II disease, per radiographs, and chronic corticosteroid use in her history to determine whether core decompression was a viable option based on direct visual inspection and load-testing of the joint surface $(5,25)$. Diagnostic hip arthroscopy in addition to traditional radiography is a method that can be used to determine the extent, type, and severity of femoral head osteonecrosis to enhance staging accuracy in early AVN, and determine appropriateness for decompression, and can be performed quickly with minimal risk. 
Stage of the disease is one parameter used to determine appropriateness for surgical decompression, as is size of necrotic lesion (less than 25\%), location of lesion (central), and intraoperative inspection is also recommended by some authors to help determine treatment in certain borderline cases $(5,23-27)$, of which our patient provided a good example.

Fig. 1: Pre-operative oblique plain radiograph of the right hip, showing no significant findings. (Or is there a slight Crescent sign on the upper portion?) 


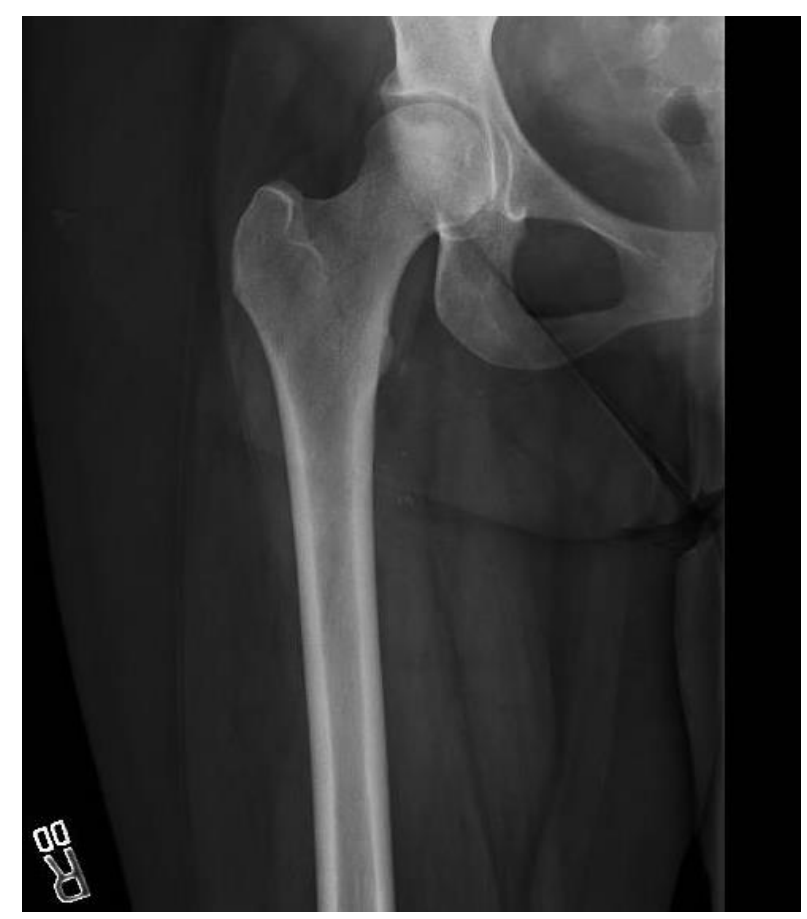

Fig. 2: Preoperative AP plain radiograph of the right hip with no significant findings.

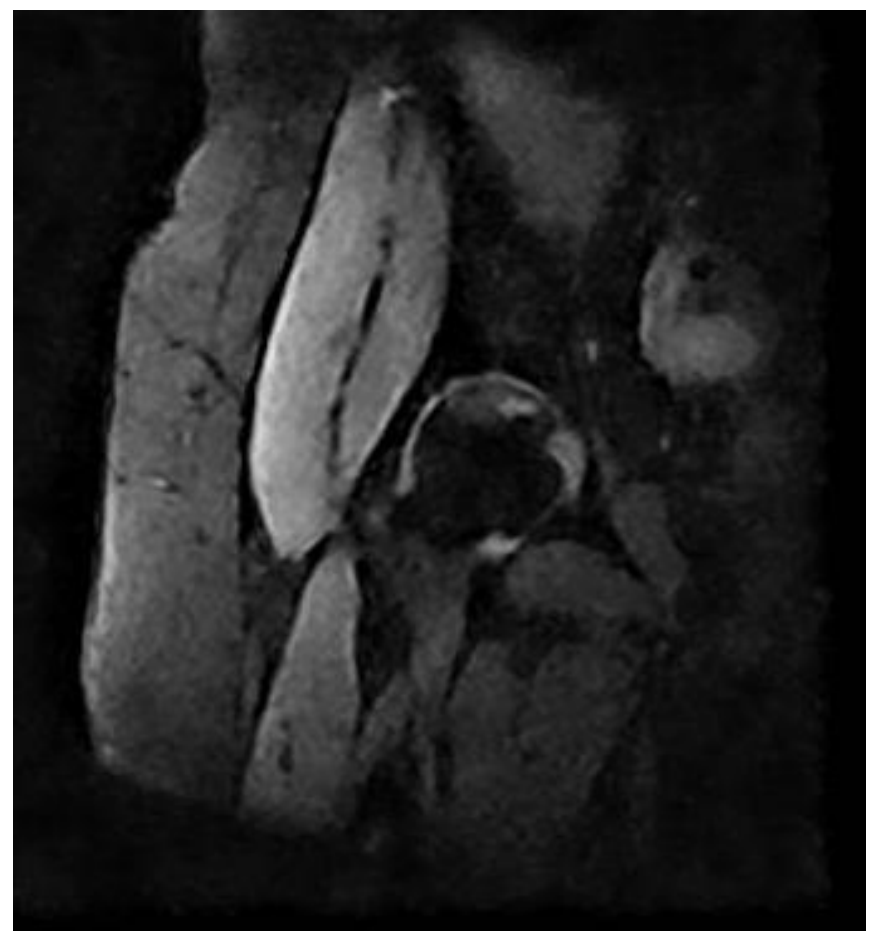

Fig. 3: Pre-surgical Coronal STIR image of the right hip showing subchondral edema of the superior weight-bearing surface, small subchondral fracture, and bone plate irregularities. 


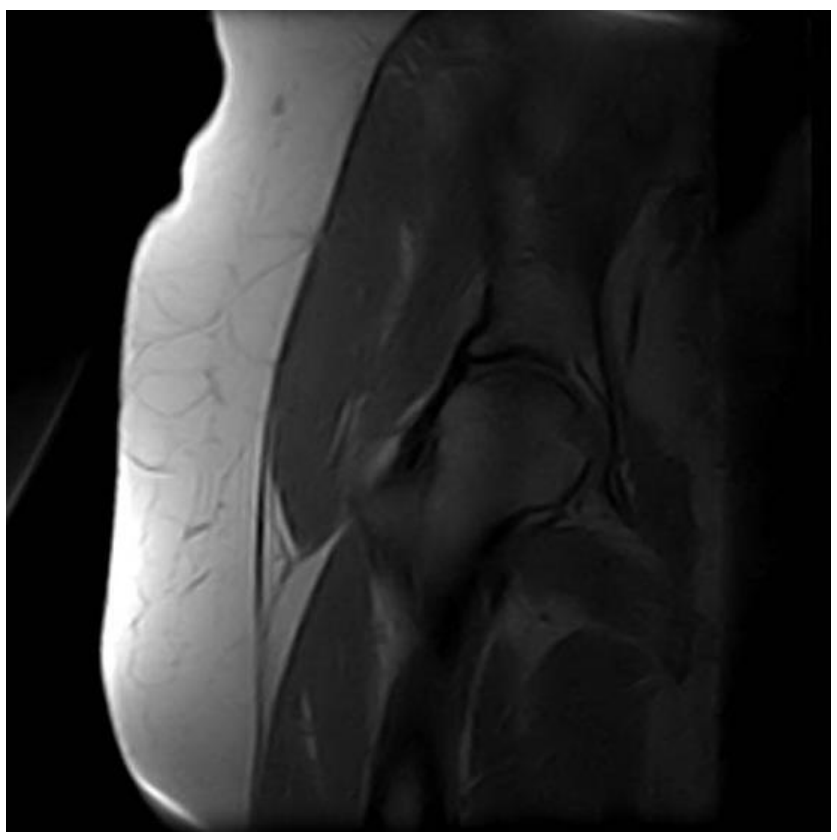

Fig. 4: T1 Weighted coronal Image showing irregular cortex and minor head depression at the posterior superior aspect.

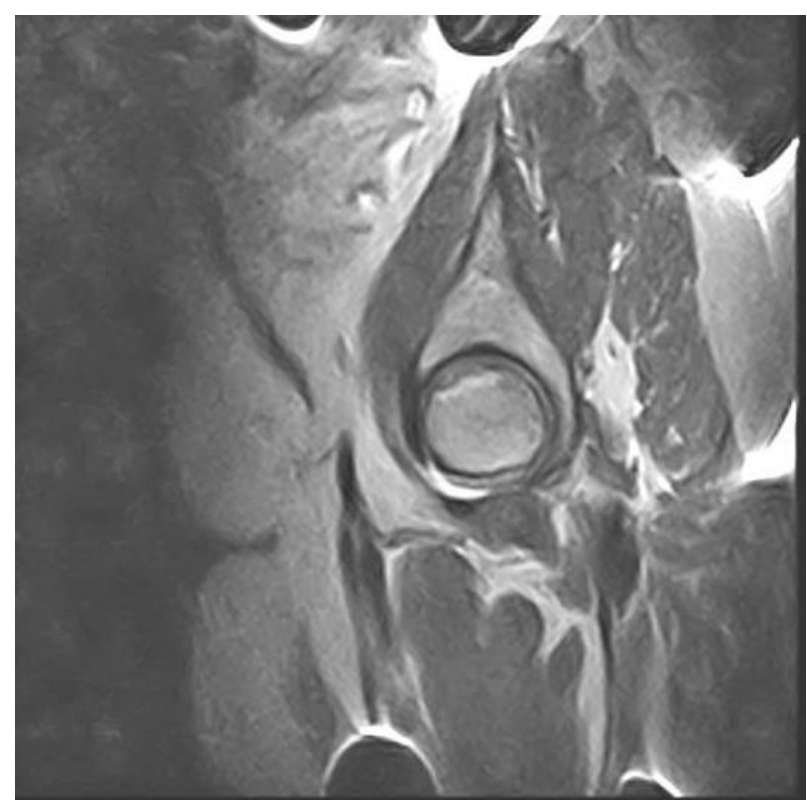

Fig. 5: MRI with sagittal spin echo showing irregular cortex and flattening of the femoral head. 


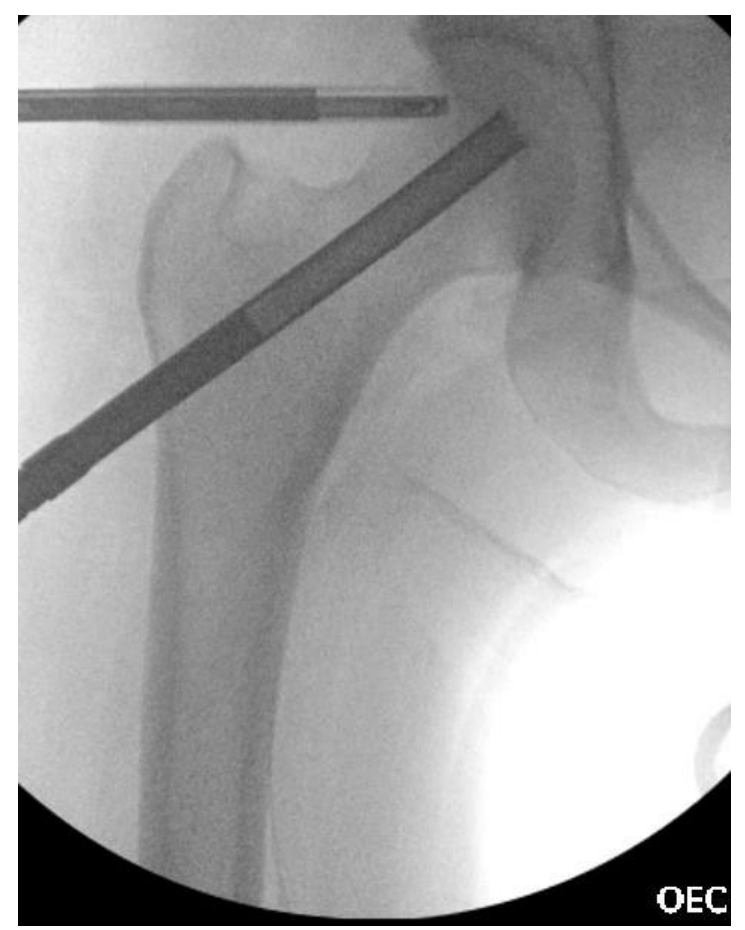

Fig. 6: Intra-operative image taken with an intensifier showing proper placement of the intramedullary cannula.

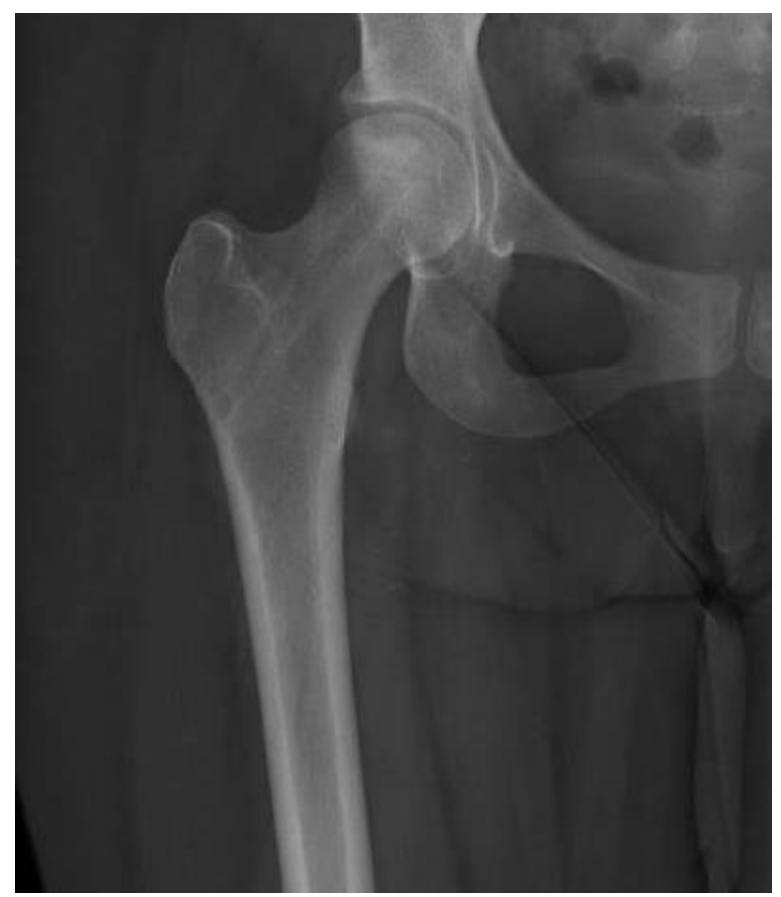

Fig. 7: Post-operative AP radiograph of the proximal femur demonstrating no further collapse of the femoral head or subchondral sclerosis. The cannulation site appears as a radio-opaque object. 